\title{
A NOTE ON THE ASYMPTOTIC EXPANSION OF A RATIO OF GAMMA FUNCTIONS
}

\author{
by JERRY L. FIELDS
}

(Received 9th December 1964)

MANY problems in mathematical analysis require a knowledge of the asymptotic behaviour of $\Gamma(z+\alpha) / \Gamma(z+\beta)$ for large values of $|z|$, where $\alpha$ and $\beta$ are bounded quantities. Tricomi and Erdélyi in (1), gave the asymptotic expansion

$$
\begin{aligned}
& \frac{\Gamma(z+\alpha)}{\Gamma(z+\beta)} \sim \sum_{j=0}^{\infty} \frac{(-1)^{j} \Gamma(\beta-\alpha+j)}{\Gamma(\beta-\alpha) j !} B_{j}^{(\alpha-\beta+1)}(\alpha) z^{\alpha-\beta-j}, z \rightarrow \infty, \\
&|\arg (z+\alpha)|<\pi, B_{0}^{(\alpha-\beta+1)}(\alpha)=1,
\end{aligned}
$$

where the $B_{j}^{(\alpha-\beta+1)}(\alpha)$ are the generalised Bernoulli polynomials, see (2), defined by

$$
\left(\frac{t}{e^{t}-1}\right)^{\sigma} e^{x t}=\sum_{j=0}^{\infty} \frac{t^{j}}{j !} B_{j}^{(\sigma)}(x),|t|<2 \pi .
$$

In this note, we show that if, instead of considering $z$ to be the large variable, we consider a related large variable, (1) can be improved from a computational viewpoint. This result is included in the following

\section{Theorem}

$$
\begin{aligned}
\frac{\Gamma(z+\alpha)}{\Gamma(z+\beta)} \sim \sum_{j=0}^{\infty} \frac{\Gamma(\beta-\alpha+2 j)}{\Gamma(\beta-\alpha)(2 j) !} B_{2 j}^{(2 \rho)}(\rho)(z+\alpha-\rho)^{\alpha-\beta-2 j}, z \rightarrow \infty, \\
|\arg (z+\alpha)|<\pi, 2 \rho=1+\alpha-\beta, B_{0}^{(2 \rho)}(\rho)=1 .
\end{aligned}
$$

Thus, the asymptotic series in (3) is essentially an even one. The proof of (3) follows directly from the loop integral representation used by Erdélyi in (1), to prove (1), i.e.,

$$
\frac{\Gamma(z+\alpha)}{\Gamma(z+\beta)}=\frac{\Gamma(1+\alpha-\beta)}{2 \pi i} \int_{-\infty \cdot e^{i \delta}}^{(0+)} e^{(z+\alpha) t}\left(e^{t}-1\right)^{\beta-\alpha-1} d t
$$

and for small $|t|$,

$$
\operatorname{Re}\left\{(z+\alpha) e^{i \delta}\right\}>0,|\delta|<\pi / 2,
$$

$$
\delta-\pi \leqq \arg \left(e^{t}-1\right) \leqq \delta+\pi .
$$

Thus (4) holds for all $\alpha$ and $\beta$ with the trivial exception $\alpha-\beta \neq-1,-2, \ldots$, and for all $z$ in the complex plane slit from $-\alpha$ to $-\alpha-\infty$. We rewrite (4) 
in the form

$$
\begin{aligned}
& \frac{\Gamma(z+\alpha)}{\Gamma(z+\beta)}=\frac{\Gamma(2 \rho)}{2 \pi i} \int_{-\infty . e^{i \delta}}^{(0+)} e^{(z+\alpha-\rho) t} t^{-2 \rho} h(t) d t, \\
& h(t)=e^{\rho t} t^{2 \rho}\left(e^{t}-1\right)^{-2 \rho}=\left[\frac{2 \sinh (t / 2)}{t}\right]^{-2 \rho},
\end{aligned}
$$

and note that if $\arg (z+\alpha)$ is fixed, and $|z+\alpha|$ taken large enough,

$$
\operatorname{Re}\left\{(z+\alpha-\rho) e^{i \delta}\right\}=\operatorname{Re}\left\{(z+\alpha) e^{i \delta}\right\}-\operatorname{Re}\left\{\rho e^{i \delta}\right\}>0 .
$$

Also, it should be noted that if $x$ is replaced by $\sigma-x$, (2) implies

$$
B_{j}^{(\sigma)}(\sigma-x)=(-1)^{j} B_{j}^{(\sigma)}(x),
$$

or

Thus,

$$
B_{2 j+1}^{(\sigma)}(\sigma / 2) \equiv 0
$$

$$
h(t)=\sum_{j=0}^{\infty} \frac{t^{2 j}}{(2 j) !} B_{2 j}^{(2 \rho)}(\rho),|t|<2 \pi .
$$

Then, since $h(t)$ is of bounded exponential growth along the path of integration, and

$$
\frac{1}{2 \pi i} \int_{-\infty . e^{i \delta}}^{(0+)} e^{z t} t^{\sigma-1} d t=\frac{z^{-\sigma}}{\Gamma(1-\sigma)}, \operatorname{Re}\left\{z e^{i \delta}\right\}>0,|\delta|<\pi / 2,
$$

Watson's lemma, see (3), is immediately applicable, and yields (3). For convenience, we note that

$$
\begin{aligned}
& B_{0}^{(2 \rho)}(\rho)=1, B_{2}^{(2 \rho)}(\rho)=-\rho / 6 \\
& B_{4}^{(2 \rho)}(\rho)=\rho(5 \rho+1) /(60), B_{6}^{(2 \rho)}(\rho)=-\rho\left(35 \rho^{2}+21 \rho+4\right) /(504)
\end{aligned}
$$

and that the $B_{2 j}^{(2 \rho)}(\rho)$ can be defined recursively by

$$
B_{2 j+2}^{(2 \rho)}(\rho)=(-2 \rho) \sum_{k=0}^{j} \frac{1}{(2 k+2)}\left(\begin{array}{c}
2 j+1 \\
2 k+1
\end{array}\right) B_{2 k+2}^{(1)}(0) B_{2 j-2 k}^{(2 \rho)}(\rho),
$$

where the $B_{2 k+2}^{(1)}(0)$ are the ordinary Bernoulli numbers defined by (2). If $\alpha$ and $\beta$ are real, and $\arg z=0$, then (3) is related to a result of Frame, see (4).

From the theorem, two corollaries are readily deduced.

Corollary 1. If $E(v)$ is a function analytic in a neighbourhood of $v=0$, $E(0)=1$, and $w$ is defined implicitly by

$$
z+\alpha-\rho=z+\frac{1}{2}(\alpha+\beta-1)=w E\left(w^{-2}\right),
$$

then there exist numbers $c_{j}$ such that

$$
\frac{\Gamma(z+\alpha)}{\Gamma(z+\beta)} \sim \sum_{j=0}^{\infty} c_{j} w^{\alpha-\beta-2 j}, z \rightarrow \infty,|\arg (z+\alpha)|<\pi, c_{0}=1 .
$$

One case of Corollary 1 is particularly interesting. If

$$
z+\alpha-\rho=w \sqrt{1+(\alpha-\rho)^{2} w^{-2}}
$$


then

$$
w=\sqrt{z(z+\alpha+\beta-1)} .
$$

Finally, we have for the hypergeometric polynomials ${ }_{2} F_{1}\left(\begin{array}{c}-n, n+\lambda \\ \beta\end{array} \mid z\right)$, see (5),

Corollary 2. If $n$ is a positive integer and $\beta$ is not a non-positive integer, then there exist numbers $c_{j}(\mu), \mu=1,2$, such that

$$
\begin{aligned}
{ }_{2} F_{1}\left(\begin{array}{c}
\left.-n, n+\left.\lambda\right|_{1}\right) \\
\beta
\end{array}\right. & =(-1)^{n} \frac{\Gamma(\beta) \Gamma(n+1+\lambda-\beta)}{\Gamma(1+\dot{\lambda}-\beta) \Gamma(n+\beta)}, \\
& \sim(-1)^{n} \frac{\Gamma(\beta)}{\Gamma(1+\lambda-\beta)} \sum_{j=0}^{\infty} c_{j}(\mu)\left(N_{\mu}\right)^{1+\lambda-2 \beta-2 j}, n \rightarrow \infty,
\end{aligned}
$$

where

$$
\begin{aligned}
& N_{1}=n+\lambda / 2, \\
& N_{2}=\sqrt{n(n+\lambda),}
\end{aligned}
$$

and

$$
c_{0}(\mu)=1, \mu=1 \text { or } 2 \text {. }
$$

\section{Acknowledgment}

This paper covers work conducted from 1st June to 1st October 1964 on research initiated by the Aeronautical Research Laboratories, Office of Aerospace Research, United States Air Force, on Contract AF 33(657)-8872, Project 7071, "Mathematical Techniques of Aeromechanics," Task 707102. The work was under the cognizance of the Applied Mathematics Laboratory with $\mathrm{Mr} \mathrm{H}$. E. Fettis acting as project director.

\section{REFERENCES}

(1) F. G. Tricomr and A. ERdélyi, The asymptotic expansion of a ratio of gamma functions, Pacific J. Math. 1 (1951), 133-142. 1924).

(2) N. E. NöRLUND, Vorlesungen über Differenzenrechnung (Springer, Berlin,

(3) G. N. WATson, A Treatise on the Theory of Bessel Functions, p. 236 (Cambridge University Press, 1958).

(4) J. S. Frame, An approximation to the quotient of gamma functions, Amer. Math. Monthly, 56 (1949), 529-535.

(5) A. Erdélyi, W. Magnus, F. Oberhettinger and F. G. Tricomi, Higher Transcendental Functions, Vol. 1 (McGraw-Hill, 1953).

Midwest Research Institute

Kansas City, Missouri 